Original Research Paper

\title{
Spousal Interaction and Breast Cancer Screening Among Korean Americans
}

\author{
${ }^{1}$ Usha Menon, ${ }^{2}$ Eunice Lee, ${ }^{3}$ Jennifer Kue, ${ }^{4}$ Arlene Miller and ${ }^{1}$ Laura Szalacha \\ ${ }^{1}$ College of Nursing, The University of Arizona, Tucson, USA \\ ${ }^{2}$ School of Nursing, University of California, Los Angeles, USA \\ ${ }^{3}$ College of Nursing, The Ohio State University, Columbus, USA \\ ${ }^{4}$ College of Nursing, Rush University, Chicago, USA
}

\author{
Article history \\ Received: 07-12-2017 \\ Revised: 07-03-2018 \\ Accepted: 30-03-2018 \\ Corresponding Author: \\ Usha Menon \\ College of Nursing, The \\ University of Arizona, Tucson, \\ USA \\ Email: umenon@email.arizona.edu
}

\section{Introduction}

Breast cancer, the most commonly diagnosed cancer among Korean American (KA) women, has had a twofold increase in incidence in the past two decades in this population (34.9 to 69.5 per 100,000) (Gomez et al., 2013). A recent study in California showed that among all Asian Americans, the greatest increase in breast cancer incidence was among Korean women (APC $=4.7$, $95 \% \mathrm{CI}=3.8,5.7)$ (Gomez et al., 2017). Mammography screening is the gold standard for early detection leading to reduction in breast cancer mortality (ACS, 2013), but despite the known benefits of mammograms, KA women have consistently suboptimal mammography rates (Lee et al., 2016; Choi et al., 2017; Chawla et al., 2015; Choi et al., 2010; Hoeffel et al., 2012). KA families retain many aspects of their cultural values and communication patterns; however, there is little to no research on family-based interventions for breast cancer screening in this population. The purpose of this study was to explore communication patterns between KA husbands and wives enrolled in a culturally-relevant, couples-based Randomized Controlled Trial (RCT) study (Lee et al., 2014) to increase mammography completion by KA wives.

Across several studies, an estimated 48 to $78 \%$ of KA women reported ever having had a mammogram in their lifetime (Lee et al., 2016; Choi et al., 2017; Chawla et al., 2015; Choi et al., 2010; Hoeffel et al., 2012; Kim et al., 2010a; Lee et al., 2009). Unfortunately, these rates are not comparable across studies because of differing operational definitions of mammography use. Importantly, however, all of these studies report screening rates for KA women below the national screening goal of $81 \%$ (USDHS, 2017). Low screening contributes to late stage breast cancer diagnosis for many KA women; thus, increasing the uptake of 
mammography use in this community is a public health priority. To this end, it is imperative to understand the influences on KA women's screening behavior and the impact that those factors can have on early detection.

There is a growing body of literature demonstrating that cultural characteristics relating to the central role of marriage, family, women's roles, self-sacrifice and the group-centered focus of Asian societies are factors that have a tremendous impact on Asian women's experiences of breast cancer and breast cancer screening (Ashing et al., 2003; Kagawa-Singer and Pourat, 2000). In many Asian cultures, even speaking among family members about breast cancer or cancer in general and the issues related to it are considered taboo (Im et al., 2000). This is attributed to the perception that breast cancer is related to issues of sexuality, as well as private parts of the body--topics that are embarrassing for Asian women to discuss openly (Lee et al., 2007; Yu et al., 2002). Asian women, in general, are also less inclined to discuss cancer and more often hide their suffering so as not to burden their families (Ashing et al., 2003). Reluctance to deal with breast health issues contributes to poor health outcomes for Asian American women and hampers their willingness to be screened (Ashing et al., 2003; Lee, 2015).

Among KA women, lower socioeconomic status, acculturation, cultural beliefs, modesty, having a Korean physician, discomfort, transportation and lack of time were all attributed to low mammography screening (Lee et al., 2014; 2016; Juon et al., 2004; Lee et al., 2006). Length of residency in the U.S. (less than 10 years) was also associated with lower mammography use (Shoemaker and White, 2016; Chawla et al., 2015). Not having health insurance also poses a major barrier to screening among KA women (Choi et al., 2017). In fact, 1 in 3 KAs lack health insurance due in part to self-employment or employment in small businesses that do not offer health insurance (APIAHF, 2015; KFF, 2008). Despite these barriers to screening, several factors have been found to positively impact KA's breast cancer screening behavior. Being married, having had a routine check-up in the past two years, being younger, having higher perceived susceptibility and benefits and lower perceived barriers (Choi et al., 2010; 2017; Eun et al., 2009; Kim et al., 2010b; Lee et al., 2006) were all significantly associated with higher mammography use.

Traditionally, the role of women in Korean society is bound to the home and family (Park and Cho, 1995). Korean women are taught at an early age to be subordinate and self-sacrificing, behaviors that are rooted in a patriarchal society (Park and Bernstein, 2008). Although this shift in traditional gender roles is changing as more KA women work outside the home and have more autonomy than previous generations, women are still pressured to uphold familial values (Park and Bernstein, 2008; Park and Cho, 1995). For example, women tend to prioritize the healthcare of the family before their own (Han et al., 2009; Park et al., 2009), which underscores the importance of empowering them to engage in early breast cancer detection behaviors. Early studies demonstrated that social support from significant others, particularly family members, is correlated with mammography utilization, especially among minority women (Gotay and Wilson, 1998; Katapodi et al., 2002; McCance et al., 1996). Interventions that included male spouses in other population groups have resulted in improved communication and listening skills and a deeper understanding of partners' cancer experiences (Duggleby et al., 2012; Jones et al., 2013). Women felt more supported by their spouse and appreciated their spouses' initiative and greater involvement in their medical care (Jones et al., 2013). KA women who perceived receiving support from family members were almost four times more likely to complete a mammogram than those who did not report such support from family members (Han et al., 2000).

Moreover, studies that examine couples' communication after diagnosis have shown that greater open communication around cancer-related issues (e.g., exchange of information, feelings and concerns) is associated with greater mutual support (Duggleby et al., 2012; Manne et al., 2006). Greater open communication was also associated with higher quality of life, better psychosocial adjustment and higher relationship functioning among couples after cancer diagnosis (Manne et al., 2006; Song et al., 2012). Husbands also play an important role in providing both emotional support (dependability, not making a fuss) and practical support (providing care) after cancer diagnosis (Emslie et al., 2009), which may be important factors in terms of supporting their wives to obtain mammography screening.

In summary, interventions to educate KA women to increase screening have not focused on leveraging the support of the Korean male spouse, or understanding the interaction between Korean spouses around mammography. Spousal support after breast cancer diagnoses has been widely studied (Chung and Hwang, 2012; Duggleby et al., 2012; Jones et al., 2013; Lewis et al., 2008). However, to our knowledge, there are no studies examining the specific role of spousal support in breast cancer prevention education other than our own work (Lee et al., 2014). Little information, however, is available about the mechanism of engaging spousal support when KA couples communicate about mammography uptake. Considering the cultural nuances of the male spousal role in the family and that spouses are a primary source of social support for married people, inclusion of spouses in preventive interventions for breast cancer is imperative.

\section{Description of the KIMCHI Program}

The sample in this study is a subset of those enrolled in a parent intervention study to increase mammography among KA women called the Korean Immigrants and 
Mammography-Culture-specific Health Intervention (KIMCHI). The KIMCHI study tested the efficacy of a couples-based intervention to increase mammogram use among non-adherent KA women (Lee et al., 2014). The intervention was designed to increase KA women's perceptions of susceptibility, seriousness of breast cancer and benefits of getting screening and decreasing barriers to mammography. The details and results of the KIMCHI trial are reported elsewhere (Lee et al., 2014). Briefly, KA religious organizations were randomized to intervention (breast cancer education) or control condition (nutrition education). Enrolled husband-wife couples completed a baseline survey, attended the educational class, completed a homework assignment and post-intervention survey at 6 and 15 months (Lee et al., 2014). Women who were randomized to the intervention condition were two times more likely to report having completed mammography at 15 months postintervention compared to those in the control condition (Lee et al., 2014). In this study, we report on communication patterns between KA spouses related to the breast cancer educational program and screening, with a focus on content and style of communication regarding the breast cancer KIMCHI intervention.

\section{Methods}

Across $50 \mathrm{KA}$ religious organizations randomly assigned to KIMCHI $(\mathrm{n}=26)$ or attention control group $(n=24)$, a total of $428 \mathrm{KA}$ couples participated in the study (Lee et al., 2014). All couples were given a homework assignment after watching an educational DVD with targeted questions on the importance of getting a routine mammogram (intervention) or improving diet (attention control group) to be completed at home within $24 \mathrm{~h}$ of receiving the education at the religious organizations. DVD use was $100 \%$ across both study groups because the DVD viewing was offered immediately post signing the consent as a group activity. To encourage completion of the homework assignment and for evidence of completion, the husband and wife were asked to write responses to at least two questions about educational program content from the DVD and at least two things that a husband could do to be supportive of his wife's breast cancer screening utilization or improving diet to be healthy. The couples were instructed to return the completed paperwork to the project office in a pre-paid envelope. Related to the primary study aims, the KIMCHI education significantly increased mammography uptake among KA women (Lee et al., 2014).

A subsample of 19 couples in the intervention group $(10 \%)$ was asked to audio-record their at-home personal interactions around the homework assignment. Notably, $100 \%$ returned the homework and recorders via mail. The audio files were transcribed verbatim in Korean and translated to English by a bicultural, bilingual assistant. A second bicultural, bilingual researcher verified accuracy of the transcripts (EL). The transcripts served as the primary data for qualitative analysis for this report.

The qualitative data were initially analyzed through inductive and iterative processes focusing on identifying major themes (Miles and Huberman, 1994; Miles et al., 2014) and domains of analysis (Strauss and Corbin, 1990). Initially, each team member independently analyzed three transcripts such that there was overlap of at least one transcript between coders. The primary codes were developed with particular attention to communication styles and the form and frequency of expression. The emerging codebook was developed through interrater consensus development. Questions about the nuances of an expression or the tone of the conversation were addressed by listening to the Korean audio-recordings as a group, led by the KA scientist (author EL), to further elicit understanding. We met over several sessions discussing independent analyses and identifying similar associations between major themes and coded segments of text. This also led to the identification of new themes not previously noted in the preliminary codebook. Interpretation disagreements were further clarified to confirm the validity of the findings (Denzin and Ye, 2000). Interrater reliability was assessed through percentage of agreement and Cohen's kappa.

\section{Results}

\section{Sample}

The 19 wives from the intervention group were, on average, 52.6 years old $(\mathrm{SD}=8.7)$ and the husbands were 57 years old $(\mathrm{SD}=10.3)$; couples had been married, on average, for 27 years $(\mathrm{SD}=3.8)$. More than half of the wives $(52.7 \%)$ had a college or graduate degree and $36.9 \%$ of the husbands had a college or graduate degree. Almost three quarters of the wives were employed (73.7) and $78.9 \%$ of the husbands were employed. On average, those who were employed worked $40 \mathrm{~h}$ a week $(\mathrm{SD}=11.2)$. Two-thirds $(68.4 \%)$ reported an income of less than $\$ 55,000$. Notably, less than half of the couples $(n=9,47.4 \%)$ had health insurance and only those who had insurance reported having a regular healthcare provider, who was, in the main, a Korean male who spoke Korean fluently. A quarter, $26.3 \%$, of the wives had a mammogram 1-2 years ago; $26.3 \%$ of the wives had a mammogram $2-3$ years ago; $21.1 \%$ of the wives had a mammogram $3-5$ years ago; $10.5 \%$ of the wives had a mammogram more than 5 years ago; and $15.8 \%$ either did not remember or had never had a mammogram. Sixteen $(68.4 \%)$ of the wives indicated that their husbands encouraged them to have a mammogram. Mean spousal support perception score for wives was 3.06 ( $\mathrm{SD}=0.48$ ) on a 5-item with 5point Likert-type spousal support instrument measuring encouragement and support received from husbands to wives, with a higher score indicating a higher level of 
support of the 19 wives in this analysis, $61 \%$ had a mammogram during the 18 month RCT $(n=11)$.

The seven emergent main themes from the qualitative analyses fell under the broad categories of interactional/discussion characteristics, cultural influences, barriers to getting a mammogram, benefits of mammogram, knowledge of breast cancer screening, strategies for getting a mammogram and reactions to the DVD (Table 1).

\section{Theme 1: Interactional/Discussion Characteristics}

Within this main theme, three clear sub-themes were discussion leader, conversation style and husband's role in wife's health.

\section{Sub-Theme: Discussion Leader}

The discussion was most often led by the husband (70\%). The conversation styles were directive, supportive, avoidant, processing and consensus building. For example, a supportive discussion included this interaction:

"...We can ask each other, "When did you receive your last [screening]? Isn't it due yet?" Just like we often check with each other about when our car is due for an oil change" (wife). An example of a processing interaction was: "...I think that is why the husband's role is very important in this regard. Husbands, including myself, should lead and encourage their spouses to have a regular examination" (husband).

A consensus-building discussion led by the husband included:

\section{"...I learned [from the DVD] that mammogram could catch tumors which are less than $1 \mathrm{~cm}$. That is why you should get it every year. How come you don't want to get it done? Is it painful? What are you afraid of?" Conversational tones differed depending on the conversation. Tones used were angry, depressed, sad, happy, resentful or blaming.}

\section{Theme 2: Cultural Influences}

Cultural influences included norms such as husbands being unaware of their wife's health habits and not discussing their wife's health. One husband said:

"Now I have realized, I feel sorry that I was really ignorant of your health matters. I should have encouraged and helped you to get the screening. I am really sorry about my ignorance in this matter" (husband). Another theme under cultural influences was found to be the focus on family. One husband, for example, stated that:

\begin{abstract}
"We have to pay more attention to family health now...pay attention to what children eat...prevent disease such as hypertension and diabetes.... we have to attend free services provided by the community organizations."
\end{abstract}

The specific and separate cultural characteristics identified in the conversations were fatalism or, in some cases, lack of fatalism, trust in God and the power of prayer. One wife stated:

“... I never really thought about breast cancer because I don't have any family members who had a breast cancer. I did not think it could happen to me. I always have prayed to God, left it up to God."

Another cultural influence was lack of preventive health orientation. One wife said:

$$
\begin{aligned}
& \text { "We, as Koreans, do not go to hospital unless } \\
& \text { we are really sick." }
\end{aligned}
$$

\section{Theme 3: Barriers to Getting a Mammogram}

Both husbands and wives identified several barriers to completing a mammogram such as fear and cost.

"I was afraid of taking a mammogram. I thought it would be so expensive to get one," said one woman. Another voiced: "I thought the whole process was too complicated." Pain was also a barrier: "Depends on the technicians who take the test, it could be very painful to take a test" and one husband noted: "...I think people are afraid of cancer, so even if they think they should get an examination, in reality, it is very hard to put into practice. It may not be easy to go to get the test alone because of [lack of] time and courage." Other barriers are listed in Table 1.

Some women felt they were not susceptible to developing breast cancer, saying:

"I should've paid more attention to my own ...but I didn't pay attention because I never thought it could happen to me." Another woman expressed: "I have been procrastinating because I thought that I would be okay. I learned today that anyone can get breast cancer and that could be a tremendous pain for all of our family." Some husbands shared this underestimation of susceptibility, saying: "I didn't think Koreans would get breast cancer as often as Europeans or Americans because Korean diet is pretty healthy..." 
Table 1: Themes and sub-themes of the husband and wife screening homework subgroup

\begin{tabular}{|c|c|c|}
\hline Main code & Sub-codes level 1 & Sub-codes level 2 \\
\hline \multirow[t]{15}{*}{ Conversation styles } & Avoidant & Pattern of speech \\
\hline & Consensus-building & Monosyllabic \\
\hline & & Talkative \\
\hline & Collaborative/not collaborative & Emotional tone \\
\hline & & Angry \\
\hline & Directive & Blaming \\
\hline & Interactive & Resentful \\
\hline & Processing type of conversation & $\mathrm{Sad}$ \\
\hline & & Depressed \\
\hline & Supportive & Dominant \\
\hline & & Giving reassurance \\
\hline & & Needing \\
\hline & & Reassurance \\
\hline & & Questioning \\
\hline & & Happy \\
\hline \multirow[t]{2}{*}{ Interactional/discussion characteristics } & Husband aware of wife's health habits/unaware & \\
\hline & Focus on family & \\
\hline \multirow[t]{6}{*}{ Cultural Influences } & Men don't usually know/discuss women's health/body issues & \\
\hline & Fatalism/lack of fatalism & \\
\hline & Trust in God & \\
\hline & Need to take care of self & \\
\hline & Power of prayer & \\
\hline & Role of religion & \\
\hline \multirow{8}{*}{ Barriers to getting screened } & Fear & Lack of risk perception \\
\hline & Lack of information & \\
\hline & Lack of perceived risk & \\
\hline & Insurance/cost & \\
\hline & Time & \\
\hline & Work & \\
\hline & Lack of courage & \\
\hline & Hard to put into practice & \\
\hline \multirow[t]{4}{*}{ Benefits of getting a mammogram } & Find cancer early & Self-care \\
\hline & Save life & Delay \\
\hline & Prevention of cancer & Commitment to health \\
\hline & Early detection & Take action \\
\hline \multirow[t]{2}{*}{ Knowledge about breast cancer screening } & Need to have regular mammogram & \\
\hline & Breast cancer risk factors & \\
\hline \multirow{3}{*}{ Strategies for getting mammogram } & Team work & Husband's role \\
\hline & Take action now & Encourage/support \\
\hline & H gives support & Joint responsibility \\
\hline \multirow[t]{8}{*}{ Reaction to DVD } & Raises awareness & \\
\hline & Increased knowledge & \\
\hline & Impressed & \\
\hline & Get screened & \\
\hline & Very beneficial & \\
\hline & Impressed & \\
\hline & Thankful & \\
\hline & Need more information & \\
\hline
\end{tabular}

Note, levels 2 and 3 can be attached to level 1 codes (i.e. 2 and 3 are not mutually exclusive from each other or from level 1)

\section{Theme 4: Benefits of Getting a Mammogram}

All of the couples indicated that they had learned much from the class and from watching the DVD. One husband remarked:

"I have learned that very unfortunate things may happen in any minute. I also learned how important it is to find breast cancer when it is in early stage." Another husband indicated: "Because we do not know what causes breast cancer, the best thing to do is to get early detection." Husbands were genuinely concerned about their spouses' health by encouraging screening so that they could live a long life. As illustrated by one husband: "I will 
make sure my wife gets yearly tests...you have to stay healthy for our family members. Let us stay healthy together."

\section{Theme 5: Knowledge of Breast Cancer Screening}

Discussions covered the need to have regular mammograms and how the DVD had emphasized that information. A second sub-theme was around breast cancer risk factors such as wives not realizing they were at risk for breast cancer.

\section{Theme 6: Strategies for Getting a Mammogram}

The strategies for getting a mammogram devised by the couples included using teamwork, specifying what the husbands' support would be and the need to take action now. A wife said to her husband:

\section{"...now you promise to pay more attention to this issue. I will make an appointment and you should help me get there." And another said: "Since I can't drive, you have to help me by driving, okay?"}

The sub-theme was to understand the husbands' role in their wives' health. One husband chimed in:

\begin{abstract}
"I will be involved with this process. I do agree with the contents of DVD that helping my wife for ride and doing chores are really necessary." Another noted: "I am going to remind you to get a mammogram and try to live a healthy life style for both of us, so that you stay positive and healthy." Other husbands were supportive in a more directive way: "You have to obey what I am telling you now. I am going to make an appointment [for you] to get all of the tests done." And another husband exclaimed: "I am living with a very brave lady."
\end{abstract}

\section{Theme 7: Reactions to the DVD}

The DVD that was the primary component of our KIMCHI educational program was the subject of much discussion, which was threaded through the conversation. Reactions included that the DVD content raised awareness and increased knowledge about breast cancer screening. They learned about the importance of prevention/early detection (get screened) from the DVD. One wife thought it (breast cancer) might be a "... disaster if not found..." Other terms used to describe the DVD were "impressed", "very beneficial" and being "thankful" for the information.

\section{Discussion}

The primary goal of the homework assignment was to supplement the KIMCHI couples' education conducted in group settings (Lee et al., 2016). In this report, we qualitatively assessed their communication specific to the intervention to enhance our understanding of the processes of communication between spouses and if that would be related to screening behavior. Clearly, the majority of husbands and wives thought the educational content of the DVD was helpful and in several cases, gave them new information about breast cancer risk and the importance of getting a mammogram. Aligned with Korean culture, which is patriarchal, husbands led the discussion $70 \%$ of the time. While we would not attempt to change such in-grained cultural behaviors, we could in future interventions focus on educating husbands about the need for screening and specific strategies to help wives.

Two cultural influences addressed were lack of preventive orientation and fatalism. The latter has been reported in African American breast cancer studies particularly (Champion et al., 2002; Rawl et al., 2002; Skinner et al., 2001; Champion et al., 2003). Barriers discussed were fear, the process being too complicated and not knowing the importance of mammography, which are in line with findings from other studies (Champion et al., 2002; Rawl et al., 2002; Skinner et al., 2001; Champion et al., 2003; Kim et al., 2016). Strategies discussed by one couple included the wife's negotiating for transportation assistance, but for the most part it was the husbands who identified ways in which they could help, such as being involved with the process, giving reminders and making appointments.

As reported previously, the couples-based intervention was successful in increasing mammography uptake (Lee et al., 2014). Based on our qualitative data analyses we speculate the KIM-CHI education influenced spousal support in ways we could not measure, which could have served as an underlying mechanism through which mammogram uptake increased (Lee et al., 2014). Additional measurement of spousal support might identify this influence more clearly and is recommended for future studies.

The DVD was well received by the study participants. The content of the DVD covered knowledge of risk and screening as well as the supportive role that husbands could play in helping their wives complete a mammogram. The actors in the DVD included the principal investigator of the primary study who is a KA nurse scientist (EL), a male KA physician, a female KA local television host and two community-based couples who described their experience of screening, being diagnosed with breast cancer and supporting each other. This analysis of focused spousal discussions after watching the DVD provides a unique picture of the interaction between KA husbands and wives around breast cancer screening. We recommend in-depth research on this concept to enhance understanding of how husbands can support their wives to get screened for 
breast cancer. One suggestion for leveraging cultural nuances in intervention development is to focus on the role of women in Korean communities. Messaging tailored on reverence of the women's role in the family and honoring health of women may improve social support from spouse and other family members.

Arguably, our findings do not encompass the entirety of communication patterns between KA wives and husbands. Our themes begin to provide an emerging picture of issues to focus on for spousal-centered educational programs.

Limitations of our study include the fact that couples in the study were in an intervention group in a larger RCT study, so some of their thoughts about breast cancer screening and the importance of spousal support may have been influenced by receiving the intervention. Because of our inclusion criterion, women in the study had not had a mammogram in the past year; their beliefs and spousal support may be different than those who are adherent with mammography.

Our findings are not generalizable for couples who live outside metropolitan areas where Korean language breast cancer screening information/services scarce resources for is not available.

\section{Conclusion}

Many interventions for immigrant minority communities are conceptualized from Western cultural perspectives. It may be hubris to assume we can change cultural norms through education. Rather, our focus must be on leveraging such norms to maximize intervention effect, such as including spouses in efforts to educate Korean women and capitalizing on the importance of interpersonal context in Korean culture. As such, our findings add to the limited literature on KAs couples' interactions about breast cancer screening. Findings will inform refinement of the original educational intervention for further testing. Our next steps include community-wide dissemination of the refined intervention with attention to cultural and environmental context, including interaction between spouses.

\section{Acknowledgments}

This study was funded by the National Cancer Institute (CA127650). We are grateful for the engagement of Korean American religious organizations, social service agencies and the study participants without whom this work would not be possible. We thank Dianna Candito and Jennifer Hulbert for providing editorial assistance.

\section{Funding Information}

This study was funded by the National Cancer Institute (CA127650). Support for authors was provided also by their institutions: The University of Arizona College of Nursing; School of Nursing, UCLA: College of Nursing; The Ohio State University, College of Nursing; Rush University

\section{Authors' Contributions}

Usha Menon: Designed study, conceptualized manuscript; analyzed data, wrote manuscript.

Eunice Lee: Designed the research; Collected and analyzed the data; and wrote sections of manuscript.

Jennifer Kue: Analyzed the data, participating in writing and revising the manuscript.

Arlene Miller: Participated in analyzin, interpreting, writing and revising the manuscript

Laura Szalacha: Conceptualized manuscript, analyzed data, wrote sections of manuscript.

\section{Ethics}

This study was approved by the Institutional Review Board (IRB) at the University of Illinois-Chicago. All consents were available in Korean and English based on the preference of the participating wives and husbands. Trained research assistants were available to answer questions about the consent prior to participants signing the consent.

\section{References}

ACS, 2013. Breast cancer facts and figures 2013-2014. American Cancer Society: Atlanta, GA.

APIAHF, 2015. http://www.apiahf.org/policy-andadvocacy/focus-areas/health-care-access.

Ashing, K.T., G. Padilla, J. Tejero and M. Kagawa-Singer, 2003. Understanding the breast Cancer experience of Asian American women. Psycho-Oncol., 12: 38-58. DOI: 10.1002/pon.632

Champion, V.L., C.S. Skinner, U. Menon, R. Seshadri and D.C. Anzalone et al., 2002. Comparisons of tailored mammography interventions at two months postintervention. Annals Behavioral Med., 24: 211218. DOI: $10.1207 /$ S15324796ABM2403 06

Champion, V., M. Maraj, S. Hui, A.J. Perkins and W. Tierney et al., 2003. Comparison of tailored interventions to increase mammography screening in non-adherent older women. Preventive Med., 36: 150-158. DOI: 10.1016/S0091-7435(02)00038-5

Chawla, N., N. Breen, B. Liu, R. Lee and M. KagawaSinger, 2015. Asian American women in California: A pooled analysis of predictors for breast and cervical cancer screening. Am. J. Public Health, 105: e98-e109. DOI: 10.2105/ajph.2014.302250

Choi, G.Y., E. Koh, S. Choi and J.Y. Cho, 2017. Understanding breast cancer screening behaviors of Korean American women in sociocultural contexts. Social Work Health Care, 56: 45-63. DOI: $10.1080 / 00981389.2016 .1244138$ 
Choi, K.S., S. Lee, E.C. Park, M.S. Kwak and B.J. Spring, 2010. Comparison of breast cancer screening rates between Korean women in America versus Korea. J. Women's Health, 19: 1089-1096. DOI: $10.1089 /$ jwh.2009.1584

Chung, C.W. and E.K. Hwang, 2012. Couples' experiences of breast cancer in Korea: A descriptive qualitative study. Cancer Nurs., 35: 211-220. DOI: $10.1097 / \mathrm{NCC} .0 \mathrm{~b} 013 \mathrm{e} 31822 \mathrm{a} 60 \mathrm{db}$

Denzin, N.K. and Y.S. Lincoln, 2000. Handbook of Qualitative Research. 2nd Edn., Sage Publications, Thousand Oaks.

Duggleby, W., J. Bally, D. C.ooper, H. Doell and R. Thomas, 2012. Engaging hope: The experiences of male spouses of women with breast cancer. Oncol. Nurs. Society, 39: 400-406.

DOI: 10.1188/12.ONF.400-406

Emslie, C., S. Browne, U. MacLeod, L. Rozmovits and E. Mitchell, 2009. 'Getting through' not 'going under': a qualitative study of gender and spousal support after diagnosis with colorectal cancer. Soc. Sci. Med., 68: 1169-1175. DOI: 10.1016/j.socscimed.2009.01.004

Eun, Y., E.E. Lee, M.J. Kim and L. Fogg, 2009. Breast cancer screening beliefs among older Korean women: Are they different from younger Korean women? J. Gerontological Nurs., 35: 40-50.

DOI: 10.3928/00989134-20090731-09

Gomez, S.L., J.V. Behren, M. McKinley, C.A. Clarke and S. Shariff-Marco et al., 2017. Breast Cancer in Asian Americans in California, 1988-2013: increasing incidence trends and recent data on breast cancer subtypes. Breast Cancer Res. Treatment, 164: 139-147. DOI: 10.1007/s10549-017-4229-1

Gomez, S.L., A.M. Noone, D.Y. Lichtensztajn, S. Scoppa and J.T. Gibson et al., 2013. Cancer incidence trends among Asian American populations in the United States, 1990-2008. J. National Cancer Institute, 105: 1096-1110. DOI: 10.1093/jnci/djt157

Gotay, C.C. and Wilson, M.E., 1998. Social support and cancer screening in African American, Hispanic and Native American women. Cancer Practice, 6: 31-7. DOI: 10.1046/j.1523- 5394.1998.1998006031.x.

Han, H.R., H. Lee, M.T. Kim and K.B. Kim, 2009. Tailored lay health worker intervention improves breast cancer screening outcomes in non-adherent Korean-American women. Health Educ. Res., 24: 318-329. DOI: 10.1093/her/cyn021

Han, Y., R.D. Williams and R.A. Harrison, 2000. Breast cancer screening knowledge, attitudes and practices among Korean American women. Oncol. Nurs. Forum, 27: 1585-91. PMID: 11103377

Hoeffel, E.M., S. Rastogi, M.O. Kim and H. Shahid, 2012. The Asian population: 2010.

Im, E.O., M.A. Hautman and B. Keddy, 2000. A feminist critique of breast cancer research among Korean women. Western J. Nurs. Res., 22: 551-570. DOI: $10.1177 / 01939450022044593$
Jones, J.M., F.M. Lewis, K. Griffith, T. Cheng and S. Secord et al., 2013. Helping her heal-group: A pilot study to evaluate a group delivered educational intervention for male spouses of women with breast cancer. Psychooncology, 22: 2102-2109.

DOI: $10.1002 /$ pon.3263

Juon, H.S., M. Kim, S. Shanka and W. Han, 2004. Predictors of adherence to Screening mammography among Korean American women. Preventive Med., 39: 474-481. DOI: 10.1016/j.ypmed.2004.05.006

Kagawa-Singer, M. and N. Pourat, 2000. Asian American and Pacific Islander breast and cervical carcinoma screening rates and Healthy People 2000 objectives. Cancer, 89: 695-705.

KFF, 2008. New analysis reveals wide disparities in health coverage among Asian Americans, native Hawaiians and Pacific Islanders, with uninsured rates for some subgroups among the highest in the United States. Kaiser Family Foundation Menlo Park, CA.

Katapodi, M.C., N.C. Facione, C. Miaskowski, M.J. Dodd and C. Waters, 2002. The influence of social support on breast cancer screening in a multicultural community sample. Oncol. Nurs. Forum, 29: 845-52. DOI: $10.1188 / 02$.ONF.845-852

Kim, J.H., U. Menon and L.A. Szalacha, 2010a. Feasibility study: Colorectal cancer related educational program for Korean Americans. Int. J. Res. Nurs., 1: 29-38. DOI: 10.3844/ijrnsp.2010.29.38

Kim, J.H., U. Menon, E. Wang and L. Szalacha, 2010b. Assess the effects of culturally relevant intervention on breast cancer knowledge, beliefs and mammography use among Korean American Women. J. Immigrant Minority Health, 12: 586-597. DOI: 10.1007/s10903-009-9246-7

Kim, K., M. Quinn, E. Chandrasekar, R. Patel and H. Lam, 2016. Partnership for healthier Asians: Disseminating evidence-based practices in AsianAmerican communities using a market-oriented and multilevel approach. JMIR Res. Protocols, 5: e123e123. DOI: $10.2196 /$ resprot.5625

Lee, E., L.F. Fogg and G. Sadler, 2006. Factors of breast cancer screening among Korean immigrants in the United States. J. Immigrant Health, 8: 223-233. DOI: $10.1007 / \mathrm{s} 10903-006-9326-2$

Lee, E., U. Menon, K. Nandy, L. Szalacha and F. Kviz et al., 2014. The effect of a couples' intervention to increase breast cancer screening among Korean Americans. Oncol. Nurs. Forum, 41: E185-93. DOI: 10.1188/14.ONF.E185-E193

Lee, E., K. Nandy, L. Szalacha, H.J. Park and K.M. Oh et al., 2016. Korean American Women and mammogram uptake. J. Immigrant Minority Health, 18: 179-186. DOI: 10.1007/s10903-015-0164-6 
Lee, E., T. Tripp-Reimer, A.M. Miller, G.R. Sadler and S.Y. Lee, 2007. Korean American women's beliefs about breast and cervical cancer and associated symbolic meanings. Oncol. Nurs. Forum, 34: 713-720. DOI: $10.1188 / 07 . O N F .713-720$

Lee, H., J. Kim and H.R. Han, 2009. Do cultural factors predict mammography behavior among Korean immigrants in the USA? J. Adv. Nurs., 65: 2574-2584. DOI: $10.1111 / \mathrm{j} .1365-2648.2009 .05155 . \mathrm{x}$

Lee, S.Y., 2015. Cultural factors associated with breast and cervical cancer screening in Korean American women in the U.S: An integrative literature review. Asian Nurs. Res., 9: 81-90. DOI: 10.1016/j.anr.2015.05.003

Lewis, F.M., B.B. Cochrane, K.A. Fletcher, E.H. Zahlis and M.E. Shands et al., 2008. Helping her heal: A pilot study of an educational counseling intervention for spouses of women with breast cancer. Psychooncology, 17: 131-7. DOI: 10.1002/pon.1203

Manne, S.L., J.S. Ostroff, T.R. Norton, K. Fox and L. Goldstein et al., 2006. Cancer-related relationship communication in couples coping with early stage breast cancer. Psychooncology, 15: 234-247. DOI: 10.1002/pon.941

McCance, K.L., K.H. Mooney, R. Field and K.R. Smith, 1996. Influence of others in motivating women to obtain breast cancer screening. Cancer Pract., 4: 141-146. PMID:8826142

Miles, M.B. and A.M. Huberman, 1994. Qualitative Data Analysis: An Expanded Sourcebook. 1st Edn., SAGE, Thousand Oaks, ISBN-10: 0803955405, pp: 338.

Miles, M.B., M. Huberman and J. Saldaña, 2014. Qualitative Data Analysis. 1st Edn., SAGE, Thousand Oaks, ISBN-10: 1452257876, pp: 381.

Park, I.H. and L.J. Cho, 1995. Confucianism and the Korean family. J. Comparative Family Stud., 26: 117-134.

Park, S.Y. and K.S. Bernstein, 2008. Depression and Korean American immigrants. Archives Psychiatric Nurs., 22: 12-19. DOI: 10.1016/j.apnu.2007.06.011
Park S., H.Y. Song, H.K. Hur and G. Kim, 2009. Effects of a cognition-oriented breast self-examination intervention for Korean women and their spouses. Public Health Nurs., 26: 259-268. DOI: $10.1111 /$ j.1525- 1446.2009.00778.x

Rawl, S., U. Menon and V. Champion, 2002. Colorectal cancer screening: An overview of current trends. Nurs. Clin. North Am., 37: 225-45. DOI: $10.1016 / \mathrm{S} 0029-6465(01) 00003-2$

Shoemaker, M.L. and M.C. White, 2016. Breast and cervical cancer screening among Asian subgroups in the USA: estimates from the national Health Interview Survey, 2008, 2010, 2013. Cancer Causes Control, 27: 825-829. DOI: $10.1007 / \mathrm{s} 10552-016-0750-5$

Skinner, C.S., V. Champion, U. Menon and R. Seshadri, 2001. Racial and education differences in mammography-related perceptions among 1,336 nonadherent women. J. Psychosocial Oncol., 20: 1-17. DOI: $10.1300 / J 077 \mathrm{v} 20$ n03 01

Song, L., L.L. Northouse, L. Z̄hang, T.M. Braun and B. Cimprich et al., 2012. Study of dyadic communication in couples managing prostate cancer: A longitudinal perspective. Psycho-Oncol., 21: 72-81. DOI: 1002/pon.1861

Strauss, A. and J. Corbin, 1990. Basics of Qualitative Research: Grounded Theory Procedures and Techniques. 17 Edn., Sage Publications, Newbury Park, ISBN-10: 0803932502, pp: 270.

USDHS, 2017. Healthy People 2020. US Dep. Health Human Services.

Yu, M.Y., A.D. Seetoo, O.S. Hong, L. Song and R. Raizade et al., 2002. Cancer screening promotion among medically underserved Asian American women: Integration of research and practice. Res. Theory Nurs., 16: 237-248. DOI: $10.1891 / 088971802780956516$ 